\title{
LA PARTICIPACIÓN E INVERSIÓN LOCAL PARA LA PROTECCIÓN DE LA CANTIDAD Y CALIDAD DEL AGUA EXPERIENCIAS EN LOS CUATRO PAÍSES ANDINOS
}

\author{
Resumen
}

Cada día va tomando mayor fuerza la necesidad de implementar esquemas de acuerdos voluntarios como estrategia para el manejo de cuencas que contribuya con la protección de la cantidad y calidad de agua en los Países Andinos (Bolivia, Colombia, Ecuador y Perú en el presente artículo).

Una de las barreras por superar en este proceso es la toma de decisiones por parte de las instituciones locales que administran el servicio del agua, en especial para consumo humano y riego, aduciendo que al implementar un mecanismo de cobro se atenta contra la población con más impuestos.

Sin embargo, en este artículo los resultados del análisis demuestran que las poblaciones de ciudades pequeñas y medianas tienen voluntad y disposición de contribuir y pagar para incentivar y compensar a propietarios de tierras en las áreas de interés hídrico de las microcuencas, mediante acuerdos o contratos para protección y restauración de ecosistemas. Adicionalmente, se demuestra también que existe un marco normativo favorable en los cuatro Países Andinos que da soporte al momento de tomar decisiones e implementar esquemas de esta naturaleza.

Con la aplicación del modelo econométrico, se logra demostrar que la disposición a pagar por protección de servicios ecosistémicos se ve mejorada en la medida que los usuarios de agua mejoren su confianza en las autoridades municipales como también dispongan oportunamente de cantidad y calidad de información ambiental.

De acuerdo al análisis de los procesos de ocho experiencias en los Países Andinos, el 61\% de usuarios tienen voluntad para pagar por la protección de cuencas, de los cuales el 90\% estaría en capacidad de pagar US $\$ 0,25$ por familia cada mes, aspecto clave para asegurar financiamiento permanente.

El marco jurídico e institucional en los Países Andinos favorece la implementación de programas con acuerdos voluntarios, a través de diferentes instrumentos como las ordenanzas y algunos mecanismos financieros como son las asignaciones presupuestarias, las tasas obligatorias y las voluntarias.

Abstract

Every day it becomes more necessary to implement voluntary agreement plans in the Andean countries as a strategy in the management of watersheds in order to contribute to the protection of quantity and quality of water.

One of the obstacles to overcome in this process is the decision making by local institutions that administer water services, especially for human consumption and irrigation, which leads to the population feeling that this payment mechanism is simply another attempt to tax them.

However, the results of the analysis presented in this article show that the populations of small and mid-sized cities are willing and ready to contribute and pay to encourage and compensate landowners in micro-watershed areas of water 
interest, by means of agreements or contracts to protect and restore ecosystems. In addition, it is also shown that there exists a favorable regulatory framework in the four Andean countries that supports making decisions and implementing plans of this type.

By applying the econometric model, it is shown that willingness to pay for the protection of ecosystem services improves as water users become more confident in the municipal authorities and if they have timely access to enough quality environmental information.

According to the analysis of eight experiences in the Andean countries, $61 \%$ of water users are willing to pay for the protection of watersheds, $90 \%$ of whom would be able to pay US\$ 0.25 per family per month, which is a key aspect to ensure permanent financing.

The legal and institutional framework in the Andean countries favors the implementation of programs based on voluntary agreements by means of different instruments, such as ordinances, and financial mechanisms, such as budget allocations and mandatory and voluntary fees.

\section{Introducción}

Actualmente va tomando mayor fuerza el principio de negociación de intereses y conflictos en una cuenca hidrográfica como estrategia básica para orientar y alcanzar resultados de conservación posibles; de alguna manera, este principio ha sido ignorado o quizás considerado poco relevante en el diseño de los planes de manejo de cuencas, puesto que la prioridad ha estado en el uso de herramientas de planificación.

La inclusión de propietarios de tierras de las áreas de interés hídrico en una cuenca, de los usuarios de agua y de las autoridades locales en un proceso de negociación nos invita a pensar y actuar de manera distinta; para un agricultor de una cuenca, la tala de árboles le interesa puesto que le permite incrementar áreas con agricultura, pero esa acción se convierte en un conflicto para quienes estamos a favor de la conservación; mientras que nuestro interés de conservar se convierte en un conflicto para ellos por el simple hecho de restringir el cambio de uso del suelo. En la medida que se logre negociar estos intereses y conflictos, se conseguirán resultados posibles y efectivos para todos. Con las autoridades y usuarios de agua se requiere comunicar, analizar y comprender estos intereses y conflictos para tomar decisiones y conseguir respaldo institucional, financiero y legitimidad en estos procesos.

Con este artículo se pretende compartir datos, información y mostrar algunas posibilidades que existen para implementar acuerdos voluntarios orientados al manejo de cuencas para proteger cantidad y calidad de agua y biodiversidad. La información compartida aquí proviene de un análisis de resultados conseguidos hasta el momento en cada experiencia, que seguro servirán de base para la reflexión al momento de tomar la decisión para entrar en este tipo de programas.

El artículo contempla tres partes: El análisis de variables que tienen relación y dependencia con la disposición a pagar en tres casos de Ecuador, el análisis de resultados sobre disposición a pagar en ocho experiencias en los Países Andinos y, finalmente, el análisis de los instrumentos jurídico-institucionales que posibilitan la toma de decisiones e implementación de mecanismos financieros para programas locales de acuerdos voluntarios en Colombia, Ecuador, Perú y Bolivia. 


\section{Objetivos}

- Determinar la dependencia y relación de variables con la disposición a contribuir/pagar por la conservación de ecosistemas en cuencas, mediante tres casos de estudio en Ecuador.

- Analizar la voluntad y disposición a contribuir/pagar por la conservación de ecosistemas en cuencas de los Países Andinos, a partir de ocho experiencias.

- Analizar los instrumentos jurídico-institucionales que en la Región Andina están facilitando la puesta en marcha de programas de acuerdos voluntarios.

\section{Revisión bibliográfica}

Los acuerdos voluntarios de conservación van ganando terreno día a día como enfoque innovador para el manejo de recursos naturales y pueden tomar diferentes denominaciones: Acuerdos Por el Agua, APA (Yaguache, 2009), Acuerdos Mutuos por el Agua, AMA (ETAPA, 2011), Arreglos Recíprocos por Agua, ARA, (rareconservation.org).

A diferencia de un esquema puro de pago por servicios ambientales (PSA) o compensación por servicios ecosistémicos (CSE) que se crearon como una opción de mercado para la compra y venta de estos servicios y como enfoque que promueve la conservación (Niesten y Rice, 2004; Hardner y Rice, 2002; Ferraro y Kiss, 2002), teóricamente para solucionar fallas de mercado puesto que prometen mayor eficiencia, eficacia y equidad en la distribución de costos y beneficios frente a los instrumentos tradicionales de comando y control, con la idea de disminuir costos e incrementar los incentivos para la innovación de prácticas (Pagiola y Platais, 2002), los acuerdos de conservación son esquemas parecidos a PSA; su diferencia está en que no se entra en la compra-venta directa de un servicio, sino en un proceso de negociación de intereses y conflictos para establecer un acuerdo o arreglo voluntario, para aplicar un mejor uso y manejo del suelo y de la cobertura vegetal que se refleje en el mejoramiento de las condiciones hidrológicas y de biodiversidad.

Según Yaguache (2009), un Acuerdo Por el Agua se desempeña bajo la participación y articulación de tres actores claves: a) los propietarios de las tierras en las áreas de interés hídrico y para biodiversidad en las microcuencas y cuencas hidrográficas; b) los usuarios de agua como familias urbanas y rurales, los usuarios de riego, las hidroeléctricas, industrias y otros tipos de usuarios y c) los administradores del servicio de agua como son las empresas municipales de agua, los municipios a través de unidades de agua potable, las juntas de regantes, las juntas de agua, los acueductos, entre otros tipos de organizaciones.

De acuerdo a Yaguache (2009), los elementos condicionantes o bases para el funcionamiento de un Acuerdo Por el Agua APA, son: el financiamiento permanente, la institucionalidad local y la adicionalidad. Se requiere asegurar recursos económicos para el financiamiento constante a través de diferentes mecanismos financieros como tasas obligatorias, tasas voluntarias, asignaciones presupuestarias y contribuciones externas. Sin una institucionalidad local, no hay garantía para la permanencia en el tiempo de estos esquemas, pues este tipo de acuerdo/enfoque es de carácter permanente por lo que se debe construir e innovar la capacidad institucional local. Por último, los acuerdos voluntarios pero condicionados que agregan 
conservación tanto en la regulación de cantidad y calidad de agua como en biodiversidad, estarían produciendo adicionalidad.

La construcción de institucionalidad para el desempeño de los acuerdos de conservación es fundamental, puesto que se requiere negociar, administrar, monitorear, evaluar e innovar los acuerdos o contratos a través de decisiones políticas y del establecimiento de instrumentos jurídico-políticos favorables. Sin embargo, se requiere trabajar ampliamente en este tema dada la vulnerabilidad institucional a la que están expuestas gran parte de las instituciones de los Países Andinos por la resistencia al cambio y a la inversión estratégica en manejo de cuencas para protección de agua.

Oportunamente, De Souza et al., (2007) abre la puerta para la innovación institucional, quien emplea como fuente filosófica, teórica y práctica, la necesidad de "cambiar las personas para cambiar las cosas y no cambiar las cosas para cambiar las personas" destacando la necesidad de construir instituciones con capacidad de manejar un conjunto de reglas, roles y arreglos institucionales que influencien prácticas y comportamientos articulados en un discurso hegemónico para alcanzar legitimidad e innovación. Resalta que el $75 \%$ de todas las iniciativas de cambio institucional fracasan en cualquier región del mundo; el $85 \%$ de los fracasos ocurre en el momento de la implementación, el 90\% de las iniciativas formulan nada más que un nuevo documento y no un nuevo comportamiento institucional, en virtud de que el 95\% de las iniciativas "cambian las cosas" para cambiar las personas, sin "cambiar las personas que cambian las cosas"; mientras que el $98 \%$ de los pocos casos de cambio institucional exitosos siguen la estrategia de "cambiar las personas" que cambian las cosas.
En este tipo de acuerdos, se espera que los usuarios contribuyan o paguen para la protección de ecosistemas y para ello es necesario conocer su disposición a pagar (DAP). Desde el concepto de la economía significa que toda persona en el momento de comprar bienes está dispuesta a pagar, a intercambiar bienes por dinero (Pearce y Turner, 1995).

La aproximación de la voluntad y disposición a pagar es un indicador de sostenibilidad financiera, puesto que muestra una opción potencial de generación de recursos financieros para las acciones de conservación y su permanencia en el tiempo. Alpizar (2006) destaca los siguientes mecanismos como fuentes de financiamiento: Impuestos, tasas retributivas, subsidios estatales, donaciones y subvenciones internacionales, pago de derechos, pagos voluntarios, mecanismos voluntarios de acreditación internacional, pagos en especie. En Brasil, en el Estado de Paraná, en 1991, se creó el impuesto ecológico del $5 \%$ del impuesto la circulación de mercancías y servicios (ICMS); en Costa Rica se asigna el 3,5\% como impuesto fijo al consumo de combustibles; en México, Conafor asigna un presupuesto anual para los esquemas de PSA; la empresa de agua potable y alcantarillado de Quito asigna el 1\% de sus ingresos al Fondo de Agua para Quito (FONAG) para ser invertidos en un fideicomiso (González et al., 2004).

En el caso de acuerdos voluntarios para protección de cuencas, varias experiencias en ejecución en los Países Andinos demuestran voluntad y disposición a contribuir/pagar, no tanto por el beneficio inmediato que esperarían recibir en el momento de iniciar el pago/ contribución, sino como inversión estratégica para asegurar la protección de ecosistemas que con los años signifique mantenery mejorar la oferta de regulación de agua. Cada programa intenta asegurar fuentes estables 
de financiamiento con la implementación de diversos mecanismos como la creación de nuevos impuestos, tasas obligatorias y voluntarias, asignaciones presupuestarias, que son los mecanismos comunes aplicados en los Países Andinos.

La disposición a pagar por lo general se ha manejado con cautela por cuanto se ha considerado como un tema social sensible de tocar por los "costos políticos que esto traería"; sin embargo, la experiencia va demostrando que la población en general, cuando se trata de contribuir por protección de agua, está dispuesta a hacerlo, mucho más si es parte de un proceso de información y con poder de participación. De alguna manera este antecedente da la pauta para desarrollar instrumentos institucionales y financieros necesarios para emprender procesos de acuerdos voluntarios.

\section{Metodología}

2.1. Estudio de la dependencia y relación de variables con la disposición a pagar en tres experiencias de Ecuador ${ }^{2}$ Para la determinación de variables que tienen relación con la disposición a pagar se realizaron tres estudios específicos en municipios de Ecuador, cuyas experiencias con acuerdos voluntarios vienen funcionando desde el año 2001 y 2005. Se empleó una muestra probabilística por cada municipio, usando el método aleatorio simple de familias usuarias del servicio de agua en los tres municipios. Se efectuaron 954 entrevistas de una pobla- ción total de 5698 usuarios de agua: 276 en Celica, 350 en Piñas y 328 en Pimampiro.

Luego de obtener la información de campo, se organizaron las variables, se ordenaron los datos y con ello se definió un modelo econométrico Logit Binario; se escogió este tipo de modelo para los tres casos pues permite trabajar con variables dependientes cualitativas tipo Dummy y también estimar la probabilidad de que la variable explicada adquiera el valor de 1. Este modelo se caracteriza porque la relación entre Pi y Xi no es lineal y también, en la medida que los valores de las variables independientes o explicativas cambian, la probabilidad de que la variable dependiente sea igual a $1 \mathrm{Pi}$ $=E\left(Y i=1 / X_{1}, X_{2} \ldots . . X_{n}\right)$ se incrementa o disminuye, pero nunca se sale de entre cero y uno. La probabilidad de aceptar el pago de los nueve centavos de dólar en función del modelo matemático expresado por Gujarati (2005), queda de la siguiente manera:

$$
\begin{gathered}
\mathrm{DAP}=1 /\left\{(1+\mathrm{e})-\left(\beta_{0}+\beta_{1} \mathrm{Y}+\beta_{2} \mathrm{IA} 1+\beta_{3} \mathrm{IA} 2+\beta_{4}\right.\right. \\
\text { IA } \left.\left.3+\beta_{5} \mathrm{CM} 1+\beta_{6} \mathrm{CM} 2+\beta_{7} \mathrm{AP}+\mu\right)\right\}
\end{gathered}
$$

Posteriormente se procesó la información relacionada con la estimación, prueba de hipótesis y verificación del modelo. Durante el procesamiento de información se eliminaron algunas variables quedando el siguiente modelo final:

$\mathrm{DAP}=1 /\left\{(1+\mathrm{e})^{-\left(\beta_{0}+\beta_{1} \mathrm{Y}+\beta_{2} \mathrm{IA} 2+\beta_{3} \mathrm{CM} 1+\beta_{4} \mathrm{AP}+\mu\right)}\right\}$

En el cuadro 1, se detallan las variables del modelo, su medición y pregunta relacionada. 


\section{Cuadro 1. Variables estudiadas}

\begin{tabular}{|c|c|c|c|}
\hline \multicolumn{3}{|c|}{ Variable } & Pregunta relacionada \\
\hline \multicolumn{4}{|c|}{ Variable dependiente } \\
\hline DAP & $\begin{array}{l}\text { Disposición a aceptar el pago de una nueva tasa: } \\
\text { nueve centavos por metro cúbico de agua con- } \\
\text { sumida en Celica, cinco centavos en Piñas y un } \\
\text { incremento de } 10 \% \text { en Pimampiro. }\end{array}$ & $\begin{array}{l}\text { Cualitativa } \\
\text { tipo Dummy } \\
\text { Si }=1, \text { No }=0\end{array}$ & $\begin{array}{l}\text { ¿Está de acuerdo con el pago de una nueva tasa ( } 9 \text { cen- } \\
\text { tavos } / \mathrm{m} 3 \text { para el año } 2009 \text { hacia adelante en Celica, } 5 \\
\text { centavos } / \mathrm{m} 3 \text { en Piñas y un incremento de } 10 \% \text { en Pi- } \\
\text { mampiro) para continuar con las actividades de conser- } \\
\text { vación de los bosques y las fuentes hídricas del cantón? }\end{array}$ \\
\hline \multicolumn{4}{|c|}{$\begin{array}{l}\text { Variables indepen- } \\
\text { dientes }\end{array}$} \\
\hline IA2 & $\begin{array}{l}\text { Información: sobre la problemática y manejo de } \\
\text { agua, que dispone la población (degradación de } \\
\text { ecosistemas, importancia del agua y correspon- } \\
\text { sabilidad de la sociedad en su manejo). }\end{array}$ & $\begin{array}{l}\text { Cualitativa } \\
\text { tipo Dummy } \\
\text { Si }=1, \text { No }=0\end{array}$ & $\begin{array}{l}\text { ¿Conoce, ha leído, escuchado u observado alguna infor- } \\
\text { mación acerca de los problemas que afectan la escasez } \\
\text { del agua? }\end{array}$ \\
\hline CM1 & $\begin{array}{l}\text { Confianza: que tiene la población en el municipio } \\
\text { (dada por el optimismo de la población hacia sus } \\
\text { autoridades por la oportuna toma de decisiones } \\
\text { e inversión estratégica en el manejo de cuencas } \\
\text { y por facilitar la participación de la sociedad civil). }\end{array}$ & $\begin{array}{l}\text { Cualitativa } \\
\text { tipo Dummy } \\
\mathrm{Si}=1, \mathrm{No}=0\end{array}$ & $\begin{array}{l}\text { ¿Confía en que el municipio toma decisiones acertadas } \\
\text { con el manejo del programa de compensación por servi- } \\
\text { cios ambientales? }\end{array}$ \\
\hline AP & $\begin{array}{l}\text { Aceptación: correspondiente a la aceptación del } \\
\text { pago actual de la tasa por servicios ambientales } \\
\text { que vienen efectuando todos los usuarios en los } \\
\text { tres cantones: cinco centavos de dólar por metro } \\
\text { cúbico en Celica, tres en Piñas y el } 20 \% \text { del pago } \\
\text { por consumo de agua en Pimampiro. }\end{array}$ & $\begin{array}{l}\text { Cualitativa } \\
\text { tipo Dummy } \\
\text { Si }=1, \text { No }=0\end{array}$ & $\begin{array}{l}\text { ¿Está de acuerdo con el pago de la tasa por protección } \\
\text { ambiental que usted viene efectuando actualmente para } \\
\text { protección de fuentes hídricas? ( } 5 \text { centavos/m3 de agua } \\
\text { consumida en Celica, } 3 \text { centavos } / \mathrm{m} 3 \text { en Piñas y } 10 \% \text { al } \\
\text { consumo de agua en Pimampiro) }\end{array}$ \\
\hline Y & Ingresos & Cuantitativa & Cuáles son sus ingresos o gastos? \\
\hline
\end{tabular}

Fuente: Yaguache (2009).

2.2. Análisis de la voluntad y disposición a contribuir/pagar Para esto, se comenzó con la revisión de seis planes de proyectos de campañas PrideARA que se vienen ejecutando desde enero de 2010 en varios municipios de los Países Andinos impulsados por organizaciones locales con apoyo de Rare Conservation, cuya información está disponible en www. rareconservation.org. Estos planes de proyecto con actividades y estrategias concretas se constituyen en el marco orientador para la ejecución de campañas enfocadas en un proceso de remoción de barreras y cambio de comportamiento, para el establecimiento de acuerdos de conservación ARA mediante estrategias de mercadotecnia social. Estas campañas involucran un trabajo participativo de dos años con audiencias de propietarios y usuarios de los servicios hídricos de las cuencas. En el cuadro 2, se detallan los seis sitios analizados (Ver página siguiente. 
Cuadro 2. Sitios analizados en los Países Andinos

$\begin{array}{lcll}\text { Institución que está liderando la experiencia } & \text { Microcuenca } & \text { Soporte Técnico } & \text { País } \\ \text { Municipalidades de San Ignacio y San José de Lourdes } & \text { Quanda } & \text { CARITAS-Jaen-RARE } & \text { Perú } \\ \text { Municipalidad de Oxapamba } & \text { San Alberto } & \begin{array}{l}\text { Instituto del Bien Común, } \\ \text { IBC-RARE }\end{array} & \text { Perú } \\ \text { Cooperativa de agua El Torno } & \text { Alto Amboró } & \text { Fundación Natura-RARE } & \text { Bolivia } \\ \text { Municipalidad de Espíndola } & \text { Jorupe } & \begin{array}{l}\text { Fundación Arco Iris- } \\ \text { RARE }\end{array} & \text { Ecuador } \\ \text { Municipalidad de Roncesvalles } & \text { Cucuana } & \begin{array}{l}\text { Fundación PROAVES- } \\ \text { RARE }\end{array} & \text { Colombia } \\ \text { Empresa de agua de San Vicente de Chucurí } & \text { Las Cruces } & \text { Fundación Natura-RARE } & \text { Colombia }\end{array}$

Fuente: rareconservation.org, Aguirre (2011), Laura (2011), Pinto (2011), Azanza (2011), Valle (2011) y Céspedes (2011).

Igualmente, se revisaron y analizaron documentos que contienen la sistematización de dos casos con varios años de experiencia en acuerdos voluntarios en Ecuador, donde está funcionando el mecanismo de cobro de tasas obligatorias a los usuarios de agua y la facilitación de incentivos y compensaciones a los propietarios de la parte alta de las microcuencas. En el cuadro 3 se describen los sitios analizados.

\section{Cuadro 3. Experiencias ecuatorianas}

\begin{tabular}{|c|c|c|c|}
\hline $\begin{array}{l}\text { Institución que está } \\
\text { liderando la experiencia }\end{array}$ & Microcuenca & Soporte técnico & Comienzo de la experiencia \\
\hline Municipalidad de Celica & Quillusara & CEDERENA & 2005 \\
\hline Municipalidad de Piñas & $\begin{array}{c}\text { Chiral, Honda, } \\
\text { Arracaches y } \\
\text { Chontas }\end{array}$ & CEDERENA & 2005 \\
\hline
\end{tabular}

Fuente: Cuenca (2008), Jaramillo (2008).

\subsection{Análisis de instrumentos jurídico-institucionales}

Se revisaron los instrumentos jurídicoinstitucionales que han facilitado la puesta en marcha de las nueve experiencias en los Países Andinos. El análisis consistió en la revisión de los instrumentos para la toma de decisiones y los mecanismos financieros implementados para cada caso, con la respectiva revisión del marco jurídico y las competencias institucionales que amparan a los municipios y empresas de agua para la administración, ejecución, seguimiento y evaluación de programas con acuerdos voluntarios. 


\section{$\ldots \ldots 3$. Resultados $\mid$ El resultado del procesamiento a través del E-Views, en la experiencia de Celica, es el \\ 3.1. Análisis de la dependencia y relación de variables con la disposición a pagar. siguiente:}

Cuadro 4. Análisis de la dependencia y relación de variables con la disposición a pagar

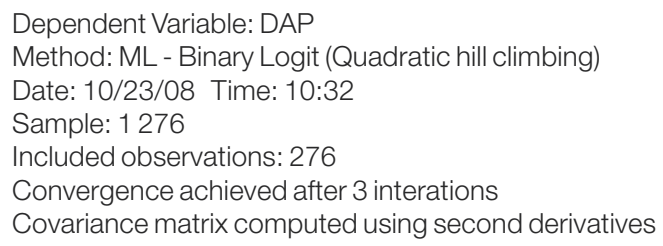

\begin{tabular}{|c|c|c|c|c|}
\hline Variable & Coefficient & Std. Error & z-Statistic & Prob. \\
\hline C & -1.991208 & 0.406227 & -4.901717 & 0.0000 \\
\hline INGRESO & 0.001299 & 0.000699 & 1.858548 & 0.0631 \\
\hline INFORMACIÓN & 0.788421 & 0.349882 & 2.253390 & 0.0242 \\
\hline CONFIANZA & 0.479948 & 0.317188 & 1.513137 & 0.1302 \\
\hline ACEPTACIÓN & 1.985768 & 0.338279 & 5.870201 & 0.0000 \\
\hline Mean dependent var & 0.605072 & \multicolumn{2}{|c|}{ S.D. dependent var } & 0.489723 \\
\hline S.E. of regression & 0.430350 & \multicolumn{2}{|c|}{ Akaike info criterion } & 1.128697 \\
\hline Sum squared resid & 50.18942 & \multicolumn{2}{|c|}{ Schwarz criterion } & 1.194284 \\
\hline Log likelihood & -150.7602 & \multicolumn{2}{|c|}{ Hannan-Quinn criter. } & 1.155016 \\
\hline Restr. log likelihood & -185.1688 & \multicolumn{2}{|c|}{ Avg. log likelihood } & -0.546233 \\
\hline LR statistic (4 df) & 68.81701 & \multicolumn{2}{|c|}{ McFadden R-squared } & 0.185822 \\
\hline Probability(LR stat) & $4.03 E-14$ & & & \\
\hline Obs with $\mathrm{Dep}=0$ & 109 & \multirow[t]{2}{*}{ Total obs } & \multirow[t]{2}{*}{276} & \\
\hline Obs with $\mathrm{Dep}=1$ & 167 & & & \\
\hline
\end{tabular}

Fuente: (Yaguache, 2009).

Las variables independientes: ingreso, información, confianza y aceptación, de acuerdo al modelo, son significativas por los valores de probabilidad cercanos a cero y explican la variable dependiente o probabilidad de aceptar el pago de la nueva tasa, cuyo cálculo se estimó en función del modelo LOGIT. 


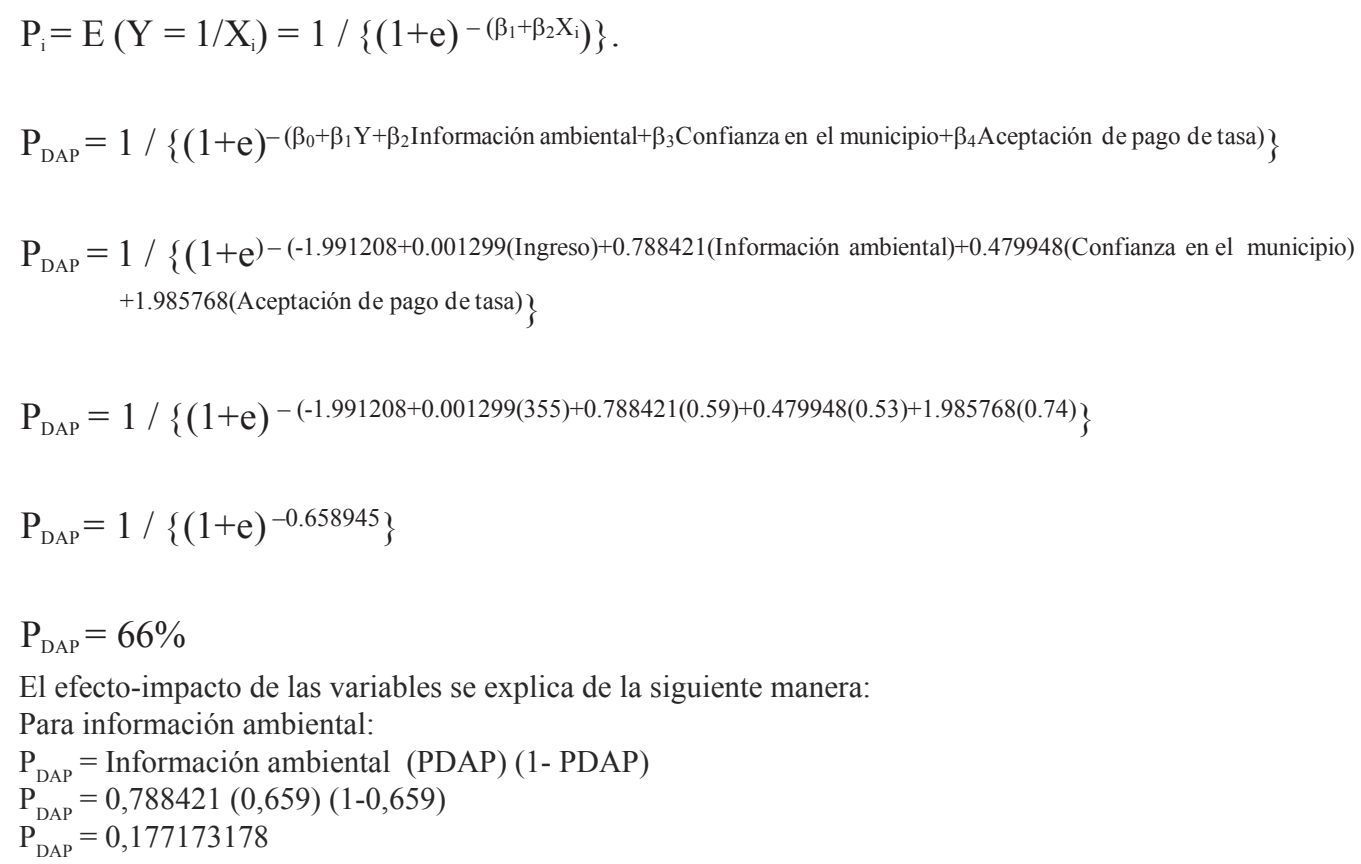

Si esta variable pasa de 0 a 1 , la probabilidad de pago $\left(P_{\text {DAP }}\right)$ se ve mejorada en $17,7 \%$; esto significa que una persona que tenga acceso a cantidad y calidad de información ambiental, tiene una probabilidad de 17,7\% más de aceptar el pago de la nueva tasa frente a una persona que no posee información.

Si la variable confianza: $P_{D A P}=$ Confianza en el municipio $\left(P_{D A P}\right)\left(1-P_{D A P}\right)$ pasa de 0 a 1 , la probabilidad de pago se ve mejorada en $10,7 \%$.

Por su parte, si la aceptación del pago de una nueva tasa: $P_{D A P}=$ aceptación de pago de la tasa $\left(P_{D A P}\right)\left(1-P_{D A P}\right)$ pasa de 0 a 1, la probabilidad de pago se mejora en $44,6 \%$.
Significa que la persona que está de acuerdo con el pago actual de la tasa tiene un 44,6\% más de probabilidad de pagar la nueva tasa de manera permanente con respecto a las personas que no aceptaron el pago.

Finalmente, frente a la posibilidad de que el ingreso: $P_{\text {DAP }}=$ Ingreso $\left(P_{D A P}\right)\left(1-P_{D A P}\right)$ mejore en $\$ 100$ dólares, la probabilidad de pago se mejora en el 2,9\%.

Independientemente, cada una de las variables también tiene su efecto-impacto sobre la probabilidad de aceptar una nueva tasa y sobre el resto de variables como se representa en el cuadro 4. 
Cuadro 5. Efecto impacto de las variables independientes

$\begin{array}{ccc}\text { Variables } & \% \\ \text { Probabilidad de pago } & \text { Real } & 65,90 \\ & 1 & 84,86 \\ \text { Información } & 0 & 17,79 \\ \text { Confianza } & 1 & 72,75 \\ \text { Aceptación } & 0 & 54,82 \\ & 1 & 70,77 \\ & 0 & 59,97 \\ & 0 & 76,41 \\ & & 30,77\end{array}$

Fuente: Yaguache (2009).

De acuerdo con el cuadro 3, al considerar que la información es 1 y el resto de variables permanecen constantes, la probabilidad de aceptar aumenta al $72,75 \%$, es decir $6,85 \%$ más que la probabilidad real (66\%); y si es 0 , la probabilidad baja al $54,82 \%$. Esto demuestra y justifica la necesidad e importancia de mantener un programa permanente de información con el propósito de sensibilizar, fomentar el intercambio de información y ganar actitud proactiva para entender la problemática y facilitar la participación y contribución de la población usuaria del agua.

Lo mismo ocurre con la variable confianza, si esta es 1 y el resto de variables permanecen constantes, la probabilidad de aceptar el pago es de 70,7\% (4,87\% más que la probabilidad real); y si es 0 , la probabilidad baja al 59,97\%. Igual con la variable aceptación del pago actual de la tasa, si el valor es de 1 y el resto de variables permanece constante, la probabilidad de aceptar la nueva tasa es de $76,4 \%$, mientras que si es 0 , esta probabilidad baja al $30,7 \%$.

Finalmente se establece que existe aceptación al programa. El rango de aceptación está dado entre el 20 y 100\% de las familias con una concentración en el 60\%; no obstante, esta cifra puede mejorar si se fortalecen las variables de confianza en el municipio y la de información ambiental, como se representa en la figura 1. 
Figura 1. Disposición a aceptar una nueva tasa

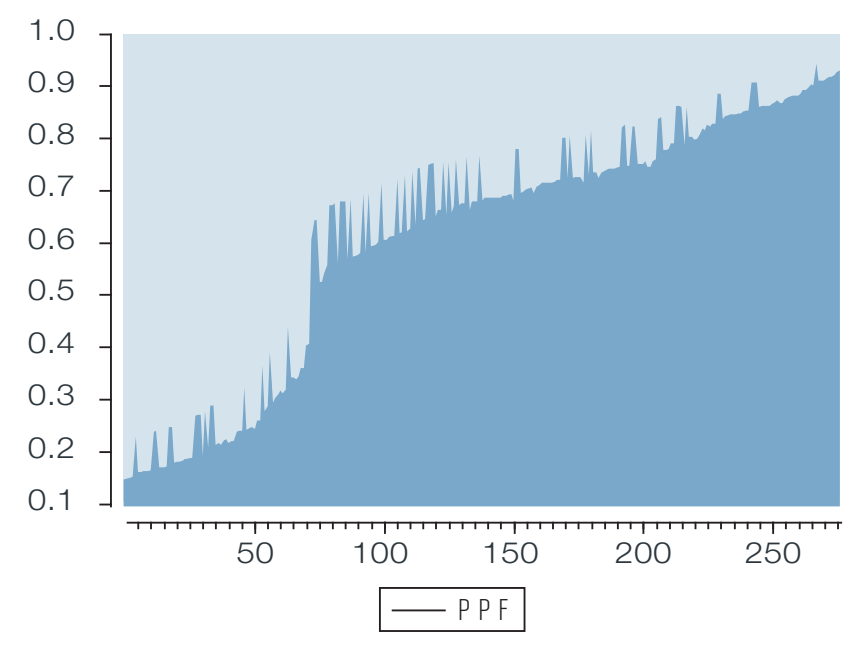

$\begin{array}{ll}\text { Forecast:PPF } & \\ \text { Aotual:PP } & \\ \text { Forecast sample:1276 } & \\ \text { Included observations:276 } & \\ & \\ \text { Root Mean Squared Error } & 0.426434 \\ \text { Mean Absolute Error } & 0.363915 \\ \text { Mean Abs. Percent Error } & 18.19577 \\ \text { Theil Inequality Coefficient } & 0.298571 \\ \text { Bias Proportion } & 0.000000 \\ \text { Variance Proportion } & 0.344575 \\ \text { Covariance Proportion } & 0.655425 \\ \text { Fuente: Yaguache (2009). } & \end{array}$

Para los casos de Piñas y Pimampiro también se corrieron modelos similares. En Piñas, los datos estadísticos del modelo son estrechos y confiables puesto que las probabilidades de aceptación de cada una de las variables son lo suficientemente pequeñas, menores al $5 \%$; la influencia de las variables independientes sobre la disposición a pagar se expresa en la siguiente correspondencia: de la variable información ambiental, en el 88\%; confianza en el municipio en el 38\%; y $66 \%$ para la variable aceptación de la tasa. Por su parte, en Pimampiro, la disposición a pagar está influenciada en un 51\% por el conocimiento sobre el programa que tienen las familias de la ciudad, su probabilidad es estrecha y confiable. En el cuadro 6 se resumen estos valores:

Cuadro 6. Influencia de las variables sobre la DAP

\begin{tabular}{cccc} 
Variable & Celica & Piñas & Pimampiro \\
$\begin{array}{c}\text { Información } \\
\text { ambiental }\end{array}$ & 17,7 & 88 & 51 \\
Confianza en el municipio & 10,7 & 38 & 70 \\
Aceptación de la tasa & 44,6 & 66 & 84 \\
\hline
\end{tabular}

Fuente: Yaguache (2009). 


\subsection{Análisis de la voluntad y disposición a pagar \\ Los resultados a la pregunta planteada en las encuestas: “¿Estaría dispuesto/a a contribuir \\ o pagar para protección de ecosistemas en cuencas?", se exponen en la figura 2.}

Figura 2. Voluntad de pago

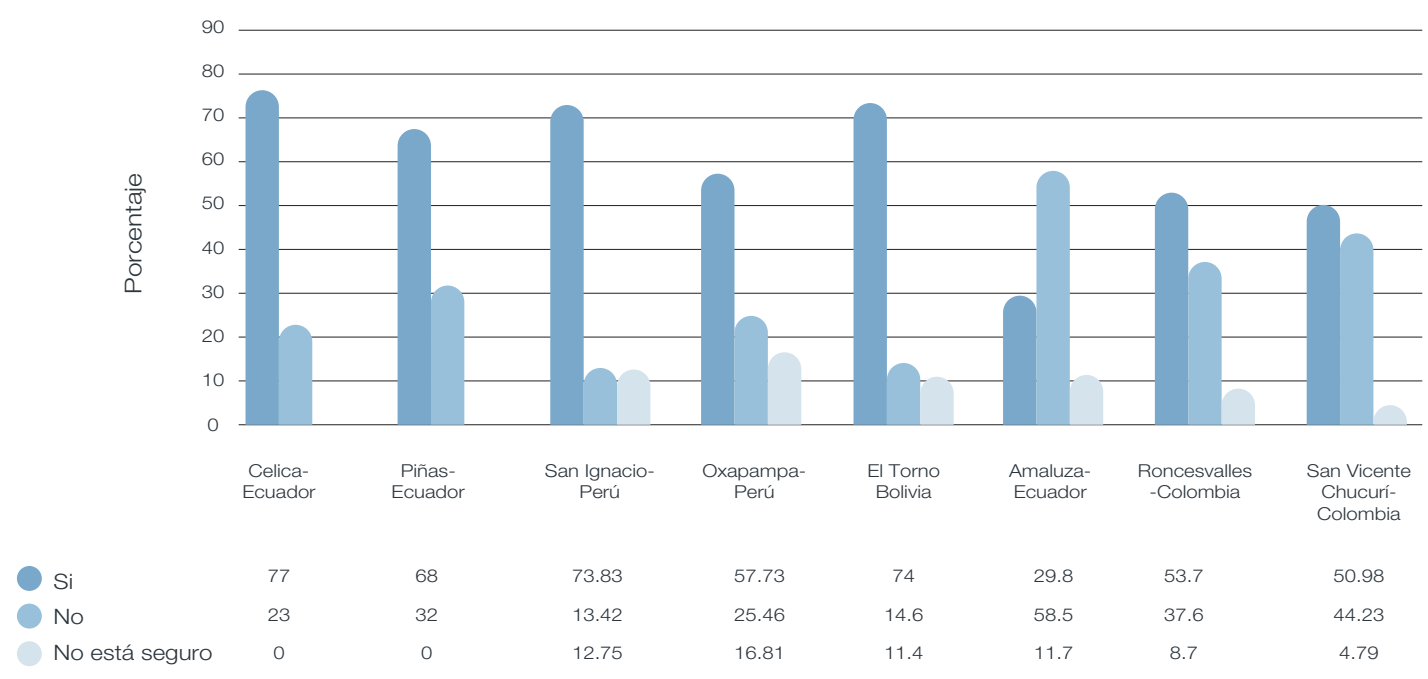

Fuente: Cuenca (2008), Jaramillo (2008), rareconservation.org, Aguirre (2011), Laura (2011), Pinto (2011), Azanza (2011), Valle (2011) y Céspedes (2011).

De acuerdo con los datos de la figura, el $61 \%$ de usuarios de agua en ciudades con poblaciones entre 5000 y 25000 habitantes tienen voluntad de contribuir o pagar económicamente para crear programas locales de acuerdos voluntarios, el $31 \%$ no está de acuerdo y un $8 \%$ no está seguro.

La voluntad demostrada en los cuatro Países Andinos es muy cercana a la voluntad para contribuir por la protección de servicios ecosistémicos en otros municipios de Latinoamérica: en el Estado de Táchira, en
Venezuela, un estudio de DAP demostró que el 73,3\% de la población está dispuesta a contribuir (Pérez et al., 2009); el 90,6\% en la ciudad de Nueva Cajamarca, Perú (Aspajo et al., 2006); y el 79\% en el municipio de Morelos, México (Brunett et al., 2010).

Al tener voluntad de pagar, se analizó la siguiente pregunta: "¿Hasta cuánto estaría dispuesto a pagar/contribuir?". Los resultados de esta información se organizan en el cuadro 7 y figura 3. 


\section{Cuadro 7. Disposición a pagar}

\begin{tabular}{|c|c|c|c|c|c|}
\hline \multirow[b]{2}{*}{ Sitios } & \multicolumn{5}{|c|}{ Disposición a pagar en dólares americanos } \\
\hline & Hasta 0.25 & $\begin{array}{c}\text { Entre } 0.25 \\
\text { y } 0.5\end{array}$ & $\begin{array}{c}\text { Entre } 0.5 \\
\text { y } 1\end{array}$ & Más de 1 & Otros \\
\hline Celica-Ecuador & 59,70 & 27,30 & 2,60 & 9,10 & 1,30 \\
\hline Piñas-Ecuador & 48,00 & 31,45 & 16,80 & 1,20 & 2,55 \\
\hline San Ignacio-Perú & 48,60 & 1,20 & 4,20 & 27,30 & 6,70 \\
\hline Oxapampa-Perú & 36,36 & 41,22 & 0,00 & 22,00 & 0,42 \\
\hline El Torno -Bolivia & 76,50 & 23,50 & 0,00 & 0,00 & 0,00 \\
\hline Amaluza-Ecuador & 31.95 & 64,85 & 0,00 & 3,20 & 0,00 \\
\hline Roncesvalles-Colombia & 24,30 & 22,96 & 15,97 & 16,80 & 19,97 \\
\hline San Vicente Chucurí-Colombia & 51,00 & 0,00 & 0,00 & 0,00 & 49,00 \\
\hline Promedio & 47.05 & 28.06 & 4.94 & 9.95 & 10 \\
\hline
\end{tabular}

Fuente: Cuenca (2008), Jaramillo (2008), rareconservation.org, Aguirre (2011), Laura (2011), Pinto (2011), Azanza (2011) Valle (2011) y Cespedes (2011).

\section{Figura 3. Disposición a pagar}

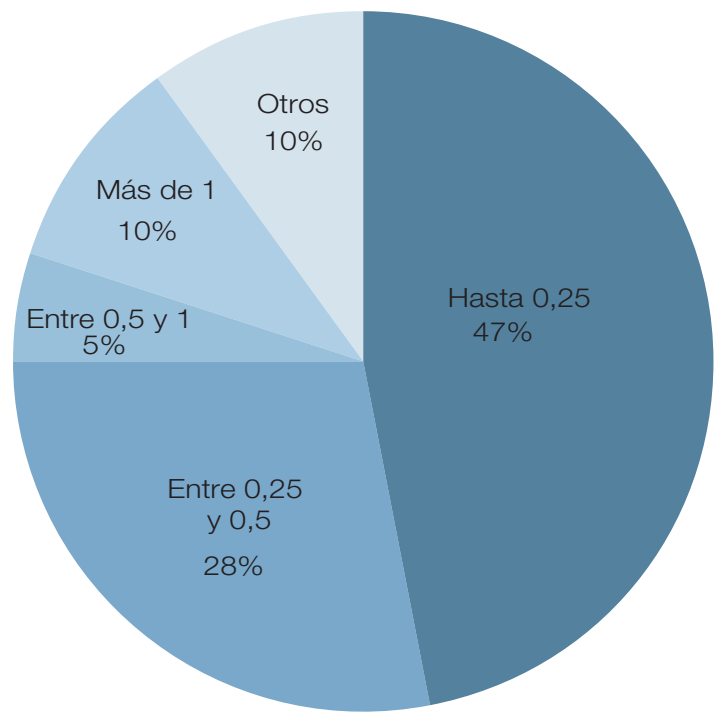

Fuente: Elaboración propia. 
De acuerdo con la figura 2, el $90 \%$ de los usuarios de agua con voluntad de pago está dispuesto a pagar hasta US $\$ 0,25$ por mes y por familia, lo que significa una tasa promedio de 1,25 centavos de dólar por metro cúbico de agua con un consumo estimado de $20 \mathrm{~m}^{3}$ de agua por familia y por mes; el 53\% está dispuesto a pagar hasta US\$ 0,5/familia/ mes una tasa aproximada de 2,5 centavos de dólar por metro cúbico; también el 25\% estaría con capacidad de pagar hasta un dólar/familia/mes/.

De alguna manera, estas tasas están dentro del rango e inclusive son menores que otras tasas en experiencias con acuerdos voluntarios implementadas anteriormente en Ecuador, en las cuales ya se están realizando los cobros, por ejemplo: Puyango cobra una tasa de US\$ 0,04/m3, US\$0,09/ $\mathrm{m}^{3}$ en Celica, US\$0,05/ $\mathrm{m}^{3}$ en Piñas, 20\% al consumo de agua en Pimampiro y US\$ 0,068/ $\mathrm{m}^{3}$ en El Chaco (Cuenca, 2008; Yaguache y Carrión 2004; Yaguache et al., 2005; JaramiIlo, 2008; Torres, 2008); muy cercanos también a valores determinados en estudios de DAP, US\$ 0,018/ $\mathrm{m}^{3}$ en Nueva Cajamarca, Perú (1 nuevo sol por familia y por mes); US\$ 0,02/ $\mathrm{m}^{3}$ en Táchira, Venezuela (hasta 1724 bolívares venezolanos por usuario y por mes bajo un consumo promedio de 20 metros cúbicos por mes y por usuario) (Pérez et al., 2009, Aspajo et al., 2006).

\subsection{Análisis de instrumentos jurídicoins-} titucionales y financieros en los Países Andinos

El marco de competencias estipuladas en las constituciones de los cuatro países permite a las municipalidades y -según su naturaleza jurídica- a las empresas de acueductos, cooperativas y empresas de agua, tomar decisiones para la creación de programas o fondos locales de manera directa o en asociación con otras instituciones, mediante la aprobación y aplicación de ordenanzas o resoluciones municipales consideradas como los instrumentos jurídicos para poner en ejecución, resoluciones en los diferentes ámbitos de desarrollo de la localidad.

En este sentido, estas ordenanzas o resoluciones han posibilitado la implementación de mecanismos para asegurar financiamiento de los programas o fondos locales que vienen funcionando en los Países Andinos, entre las que se destacan: las tasas obligatorias y voluntarias para protección de fuentes de agua y las asignaciones presupuestarias.

A continuación se detallan los elementos y características de estos instrumentos financieros:

\section{a) Las tasas obligatorias}

Una tasa es una especie de tributo que se impone, en este caso, por la protección de fuentes hídricas/cuencas; la característica esencial es que es de carácter permanente y cada uno de los usuarios está en obligación de pagarla una vez que se toma la decisión por el concejo municipal o empresa de agua, previo un estudio de disposición a pagar, realización de una audiencia pública, o por resolución de asamblea general de socios de las empresas de acueductos o cooperativas de usuarios de agua. Si un programa local de acuerdos voluntarios contempla el manejo de incentivos y compensaciones anuales, entonces el financiamiento con este tipo de instrumento es el más prometedor, porque se asegura el pago mensual o anual de manera permanente.

La implementación de este tipo de tasas es posible encontrarla en Ecuador, Perú y Bolivia bajo el siguiente soporte jurídico:

En Ecuador, la Constitución de la República (www.asambleanacional.gov.ec) en su artículo $N^{\circ} 264$ faculta a los gobiernos municipales competencias exclusivas, espe- 
cíficamente en los literales: 1) "Planificar el desarrollo cantonal y formular los correspondientes planes de ordenamiento territorial, de manera articulada con la planificación nacional, regional, provincial y parroquial, con el fin de regular el uso y la ocupación del suelo urbano y rural; 2) Ejercer el control sobre el uso y ocupación del suelo en el cantón; 3) Prestar los servicios públicos de agua potable, alcantarillado, depuración de aguas residuales, manejo de desechos sólidos, actividades de saneamiento ambiental y aquellos que establezca la ley; 4) Crear, modificar o suprimir mediante ordenanzas, tasas y contribuciones especiales de mejoras".

Además, el Código Orgánico de Organización Territorial Autonomía y Descentralización de Ecuador COOTAD (www.asambleanacional.gov.ec) faculta funciones a los municipios a la hora de tomar decisiones para el establecimiento de este tipo de programas. Específicamente en el artículo $N^{\circ} 54$, en los siguientes literales: a) "Promover el desarrollo sustentable de su circunscripción territorial cantonal, para garantizar la realización del buen vivir a través de la implementación de políticas públicas cantonales, en el marco de sus competencias constitucionales y legales; [...] e) Elaborar y ejecutar el plan cantonal de desarrollo, el de ordenamiento territorial y las políticas públicas en el ámbito de sus competencias y en su circunscripción territorial, de manera coordinada con la planificación nacional, regional, provincial y parroquial, y realizar en forma permanente, el seguimiento y rendición de cuentas sobre el cumplimiento de las metas establecidas; [... k) Regular, prevenir y controlar la contaminación ambiental en el territorio cantonal de manera articulada con las políticas ambientales nacionales".

En Perú, la Superintendencia Nacional de Servicios de Saneamiento (SUNASS) tiene la competencia para aprobar fórmulas y estructuras tarifarias. Para la empresa de servicios públicos de Moyobamba, la SUNASS aprobó un incremento tarifario, equivalente a una tasa, cuyos recursos se destinan al proyecto de mejoramiento de la cantidad y calidad del recurso hídrico, amparados en el artículo N 31 del Reglamento de la Ley General de Servicios de Saneamiento (SUNASS, 2007).

Por su parte en Bolivia, la Ley de Municipalidades No 2028 del 28 de octubre de 1999 (http://www.legislacionmunicipal.fam.bo) atribuye competencias a los municipios así: "Art. 4.II.2. La facultad de generar, recaudar e invertir recursos; [...] Art. 4.II.3 La potestad de dictar ordenanzas y resoluciones determinando así las políticas y estrategias municipales; [...] Art. 5.II.4. Preservar y conservar, en lo que corresponda, el medio ambiente y los ecosistemas del Municipio, contribuyendo a la ocupación racional del territorio y el aprovechamiento sostenible de los recursos naturales".

Gran parte de las cooperativas de usuarios de agua de Bolivia son autónomas y mediante asamblea general de socios están en capacidad de aprobar e implementar tasas obligatorias para protección de fuentes de agua.

\section{b) Tasas voluntarias}

Este tipo de tasa se aplica en los cuatro países, pero en Colombia se está implementando como un instrumento para el recaudo de las aportaciones voluntarias de los usuarios de agua de consumo humano como alternativa a una tasa obligatoria que, de acuerdo al marco normativo vigente, no es posible implementar en este país, a diferencia de Perú, Ecuador y Bolivia. Estas tasas voluntarias funcionan con un cupón desprendible adjunto a la planilla de pago por consumo de agua u otros servicios públicos. 


\section{c) La asignación de recursos}

En los cuatro países funciona la asignación de recursos de los presupuestos corrientes como estrategia de financiamiento. Para esto, las resoluciones de concejos municipales o asambleas de usuarios se constituyen en los instrumentos adecuados para la toma de decisiones.

Colombia frente a Ecuador, Bolivia y Perú, tiene la ventaja que por ley se faculta a los municipios a invertir, de acuerdo a la Ley $\mathrm{N}^{\circ}$ 99 de 1993, (www.oas.org/dsd/fida/laws/ legislation/colombia) a través de su artículo $\mathrm{N}^{\circ} 111$, posteriormente modificado por el artículo $N^{\circ} 210$ de la Ley $N^{\circ} 1450$ (www.secretariasenado.gov.co/senado/.../ ley/2011/ley_1450_2011.ht...) en la adquisición de tierras en cuencas que abastecen de agua a los acueductos, y en esquemas de pago por servicios ambientales. Los recursos para estas actividades provienen de los ingresos por transferencias que el sector eléctrico está en la obligación de hacer de acuerdo con el artículo $\mathrm{N}^{\circ} 45$ de la misma Ley N 99 de 1993.

En otro contexto, la empresa del acueducto de Queremal, Cali, Colombia, reformó sus estatutos en asamblea general de socios para aprobar que los excedentes del manejo financiero se inviertan en el manejo de la microcuenca San Juan, la misma que abastece de agua a este poblado (www.rareconservation.org).

\section{Conclusiones}

- Lo que se logra con la aplicación del modelo econométrico Logit, en los tres municipios de Ecuador, es demostrar que existen dos variables claves que influyen en la voluntad y disposición a pagar para el sostenimiento de programas locales de acuerdos voluntarios: o La cantidad y calidad de información disponible para usuarios de agua, para los propietarios de las tierras en las microcuencas, así como también para las autoridades locales encargadas de tomar decisiones.

o La confianza que los usuarios de agua o población en general tienen en sus autoridades por las decisiones oportunas, la inversión estratégica para el manejo de cuencas y la innovación de programas locales.

o Por su parte, tanto el ingreso como la aceptación del pago actual de la tasa no tienen mayor influencia sobre la disposición a pagar.

- De acuerdo con los resultados encontrados del análisis de disposición a pagar en las experiencias de Países Andinos, se evidencia un escenario favorable y posible para crear tasas o asignar recursos para conformar fondos locales con capacidad de mantener financiamiento permanente como un pilar fundamental para el sostenimiento de los programas de acuerdos voluntarios. La voluntad y disposición a pagar tasas por parte de usuarios de agua de consumo doméstico se refleja en más del $61 \%$ de la población con una tasa mensual entre US\$0,25 y 0,5 por familia.

- Los resultados de esta corta investigación demuestran que existen condiciones institucionales, legales y financieras para tomar decisiones y con ello crear fondos o programas locales de acuerdos voluntarios. En gran parte de municipios o empresas de agua es muy común escuchar a sus autoridades que una decisión de esta naturaleza es un impuesto más para los ciudadanos; sin embargo, las 
experiencias analizadas demuestran que la población está dispuesta a apoyar y a pagar, mucho más cuando se sientan parte de un proceso de información, sensibilización y participación. Más allá de que la creación de un fondo/programa local tenga un costo político, es una oportunidad estratégica de inversión para municipios, empresas, acueductos y cooperativas de agua.

- Los municipios de la Región Andina interesados en implementar programas locales de esta naturaleza deberían tomar contacto con estas experiencias de los cuatro países, para conocer el proceso vivido y los principales aprendizajes de manera que puedan extraer los elementos claves para ser adaptados de acuerdo a cada uno de sus contextos particulares.

- Se requiere desarrollar programas permanentes de comunicación con la finalidad de facilitar información oportuna para sensibilizar a los diferentes tipos de familias y comunidades, facilitando un proceso de cambio de actitud y comportamiento hacia la protección de eco- sistemas asumiendo su responsabilidad como ciudadanos, con la posibilidad de crear poder local para apoyar y legitimar los procesos de acuerdos voluntarios.

- Una estrategia clave en este proceso de información es la metodología "Pride" que ejecuta Rare Conservation a través de "campañas orgullo" para impulsar el cambio de comportamiento; ahora se encuentra ejecutando varias campañas en los Andes para protección de cuencas y especies en peligro de extinción con resultados, hasta el momento, muy importantes para conseguir oportunamente decisiones de autoridades, disposición a pagar por parte de usuarios y la firma de acuerdos de conservación con los propietarios de las tierras de las partes altas. La metodología Pride, maneja una teoría de cambio basada en la facilitación de información para provocar una actitud proactiva y comunicación interpersonal que conlleven a remover barreras para cambios de comportamiento los cuales se reflejen en la reducción de amenazas de los ecosistemas y con ello conseguir los resultados de conservación propuestos. 


\section{REFERENCIAS BIBLIOGRÁFICAS}

Aguirre H. (2011) Plan de proyecto de la campaña Orgullo en Quanda. Rare Conservatión, Caritas. Jaén, Perú.

Alpízar F. (2006) Bienes y servicios ambientales, mercados no tradicionales, mecanismos de financiamiento y buenas prácticas en América Latina y El Caribe. Centro Agronómico Tropical de Investigación y Enseñanza, Banco Interamericano de Desarrollo, Diálogo regional de política. Costa Rica.

Aspajo F., López F., Vásquez P. y Zumaeta A. (2006) Disponibilidad a pagar por servicio ambiental hídrico en la ciudad de Nueva Cajamarca, subcuenca del Yuracyacu y Avisado. Empresa Pública de Servicios de Moyobamba. Proyecto Regional de cuencas Andinas. GTZ. Perú.

Azanza M., (2011). Plan de proyecto de la campaña Orgullo en Jorupe. Rare Conservatión, Arco Iris. Loja, Ecuador.

Brunett E., J. Baró, E. Cadena y Esteller María M. (2010) Pago por servicios ambientales hidrológicos, caso de estudio Parque Nacional del Nevado de Toluca, México.

Céspedes C. (2011). Plan de proyecto de la campaña Orgullo en Las Cruces. Rare Conservatión, Fundación Natura Colombia. San Vicente de Chucurí. Colombia.

Cuenca J. (2008) Protección de la cantidad y calidad del agua. La experiencia del municipio de Celica. Corporación para el Desarrollo de los Recursos Naturales, CEDERENA. Loja. Industria Gráfica Amazonas. Ecuador.

De Souza J., J. Santamaría, J. Cheaz, A. Mato, S. Valle, A. Gomes, A. Maestrey, F. Álvarez, J. Ordóñez, N. Rodríguez, M. Chi liquinga y N. Dolberg (2007) ¿Quo Vadis, Transformación Institucional? La innovación de la innovación, del cambio de las cosas al cambio de las personas que cambian las cosas. Instituto Internacional de Investigación sobre Políticas Alimentarias (IFPRI) Red Nuevo Paradigma para la Innovación Institucional en América Latina División ISNAR. Costa Rica.

ETAPA (2011). Plan de Proyecto, campaña Orgullo. Empresa Pública de telecomunicaciones, agua potable, alcantarillado y saneamiento. Cuenca, Ecuador.

Ferraro P. \& Kiss A. (2002). "Direct payments for biodiversity conservation", en Science, 298, pp. 1718-1719.

González M., A. Aldrete, A. Guerrero, E. Telles, De los Santos H., Benavides J., Hernández J., Lazalde R., De la Rosa P. y Cazares S. (2004) Experiencias sobre mecanismos de pago por servicios ambientales hidrológicos: Análisis comparativo en varios países de América. Estudio de caso. Comisión Nacional Forestal CONAFOR. Postgrado forestal. Colegio de Postgraduados. México.

Gujarati Damodar (2005). Econometría. México DF. McGraw-Hill Interamericana Editores. Cuarta edición. México.

Hardner J. \& R. Rice (2002). "Rethinking green consumerism", en Scientific American May: 89-95.

Jaramillo C. (2008). Protección de la cantidad y calidad del agua. La experiencia del municipio de Piñas. Corporación para el Desarrollo de los Recursos Naturales, CEDERENA. Loja. Industria Gráfica Amazonas. 


\section{Ecuador.}

Laura C. (2011). Plan de proyecto de la campaña Orgullo en San Alberto. Rare Conservatión, IBC. Oxapampa. Perú.

Niesten E \& R. Rice (2004). "Sustainable forest management and conservation incentice agreements", en International Forestry Review. 6:56-60.

Pagiola S. y G. Platais Gunars (2002). "Pago por servicios ambientales", en Environment Strategy Note № 3 , Banco Mundial. Washington DC.

Pearce D. y K. Turner (1995). Economía de Los Recursos Naturales y del Medio Ambiente. España. Edigrafos, S.A.

Perez José, Naranjo María Elena y Henao Ángela (2009). Determinación de la disposición a pagar por el servicio ambiental protección de los recursos hídricos en el estado de Táchira, Venezuela. Revista Técnica de Ingeniería Universidad de Zulia abril 2009, vol.32, no 1.

Pinto P. (2011). Plan de proyecto de la campaña Orgullo en El Torno. Rare Conservatión, Fundación Natura Bolivia. Santa Cruz, Bolivia.

Superintendencia Nacional de Servicios de Saneamiento (2007). Reglamento general de regulación tarifaria. Normas legales, Resolución de Consejo Directivo No. 009-2007. Lima. SUNASS-CD. Febrero de 2007. Perú.

Torres M. (2008). Protección de la cantidad y calidad del agua. La experiencia del municipio de Puyango. Corporación para el Desarrollo de los Recursos Naturales, CEDERENA. Loja. Industria Gráfica Amazonas. Ecuador.

Valle H. (2011) Plan de proyecto de la cam- paña Orgullo en Roncesvalles. Rare Conservatión, Proaves. Roncesvalles. Colombia.

Yaguache R. (2009) Análisis económico del Acuerdo Por el Agua. Estudio de caso, Municipio de Celica, Ecuador. Tesis para obtener el grado de Doctor en Economía de Los Recursos Naturales y Desarrollo Sustentable. Facultad de Economía, Universidad Nacional Autónoma de México. México DF.

Yaguache R. y R. Carrión (2004). Construyendo una experiencia de desarrollo. El manejo de recursos naturales en Pimampiro. Corporación para el Desarrollo de los Recursos Naturales, CEDERENA. Quito. Soboc Grafic. Ecuador.

Yaguache R., D. Domínguez, R. Carrión y E. Zarria (2005). La experiencia del cantón El Chaco en la protección de sus fuentes de agua. Ministerio del Ambiente, Banco Interamericano de Desarrollo. Quito. Soboc Grafic. Ecuador.

Páginas web www.asambleanacional.gov.ec www.legislacionmunicipal.fam.bo www.oas.org/dsd/fida/laws/legislation/ colombia www.secretariasenado.gov.co/senado/.../ ley/2011/ley_1450_2011.ht... 